\title{
Analytical relationships between elliptic accelerating cavity shape and fields
}

\author{
Valery Shemelin* and Georg Hoffstaetter \\ Cornell Laboratory for Accelerator-based Sciences and Education (CLASSE), Ithaca, \\ New York 14853, USA
}

(Received 20 May 2014; published 24 October 2014)

\begin{abstract}
Here we describe some relationships between cavity shape and fields on and near its surface that can be used for better understanding of the surface field properties. The problem of accelerating cavity optimization lies in the search of the shape with minimal peak magnetic or electric field for a given acceleration rate. This problem became especially important due to widespread use of superconducting cavities where the maximal magnetic field appeared to have a hard limit. The peak magnetic field can be lowered if one can increase the peak electric field but the electric field is also limited because of field emission. The problem of minimal losses in a cavity is very close to the problem of minimal peak magnetic field, though it is not the same, it relates to the lowest average field for a given acceleration rate. The field configuration on the cavity surface is also responsible for the phenomenon of multipactor. Cavities with these properties-minimal peak fields, minimal losses, and absence of multipactor-are found within the set of elliptic cavities. Further improvement of these properties is possible if we step out of the limits of elliptic shapes.
\end{abstract}

DOI: 10.1103/PhysRevSTAB.17.102001

PACS numbers: $84.40 .-\mathrm{x}, 85.25 . \mathrm{Am}$

\section{INTRODUCTION}

The commonly used superconducting cavity shape for high $\beta$ values is a result of evolution from a pillbox rf cavity with the beam tubes added and rounded walls - to decrease the peak electric field - to a shape consisting of elliptic arcs to prevent multipacting [1,2]. One of results of this evolution is the reentrant elliptic cavity that has now a record achieved acceleration rate $[3,4]$. The relationship between the parameters of these arcs is a subject of cavity optimization in search of the best consistency between maxima of the surface electric and magnetic fields that defines the maximal acceleration achievable in the cavity. All other figures of merit of a cavity also depend on the arc parameters but either they are defined by different requirements, e.g. aperture radius should be big enough to reduce the wakefields, or are connected with low magnetic fields: wall losses are practically minimal for a cavity optimized for lowest peak magnetic field [5].

In this paper, we present some relationships found for the fields in an elliptic cavity defined by its shape. These relations can be used for any axially symmetric cavity with a smooth inner surface and would be useful in search of an other than elliptic shape with minimal peak magnetic field, or, more definitely, with minimal value of $H_{\mathrm{pk}} / E_{\mathrm{acc}}$ for given values of $E_{\mathrm{pk}} / E_{\mathrm{acc}}$, wall slope angle $\alpha$, and the

*vs65@ cornell.edu

Published by the American Physical Society under the terms of the Creative Commons Attribution 3.0 License. Further distribution of this work must maintain attribution to the author $(s)$ and the published article's title, journal citation, and DOI. aperture radius $R_{a} . H_{\mathrm{pk}}$ and $E_{\mathrm{pk}}$ are peak magnetic and electric fields; $E_{\text {acc }}$ is the accelerating field.

As it was shown in [5] and [6], if these three parameters are given: $E_{\mathrm{pk}} / E_{\mathrm{acc}}, \alpha$, and $R_{a}$, the minimal value of $H_{\mathrm{pk}} / E_{\mathrm{acc}}$ for the elliptic shape can be found. If no limitations are used for the wall slope angle $\alpha$, we will come to the reentrant shape having the minimal $H_{\mathrm{pk}} / E_{\mathrm{acc}}$ from all possible for given $E_{\mathrm{pk}} / E_{\mathrm{acc}}$ and $R_{a}$ [3]. It is worthy to note that $H_{\mathrm{pk}} / E_{\mathrm{acc}}$ is a monotone function of any of these three parameters: it decreases for smaller $\alpha$ and $R_{a}$, and for bigger $E_{\mathrm{pk}} / E_{\mathrm{acc}}$.

Any elliptic cavity can be found on the graphs analogous to those presented in [5] and [6]. So, the further search of new shapes should be done for nonelliptic cavities.

We have to make the reservation that no shape different from elliptic is presented in this paper. However, we found some new relationships between cavity shape and its surface fields. We also found Taylor expansions of the fields in the most important places of the cavity: near the equator and near the iris tip, where the magnetic and the electric field, respectively, are maximal. These expansions of fields near the equator were used for multipactor calculations [7].

\section{ELLIPTIC GEOMETRY AND SURFACE FIELDS}

All earlier optimizations for minimal $H_{\mathrm{pk}} / E_{\mathrm{acc}}$ were done under the assumption that the components of the shape are elliptic arcs connected with a straight segment. Figure 1 shows one half-cell, i.e. one element of periodicity.

As an example, three widely known geometries of the inner half-cells are shown in Fig. 2: TESLA [8] from DESY, the low-loss cavity [9] from JLab, and the reentrant cavity by Cornell [3]. 


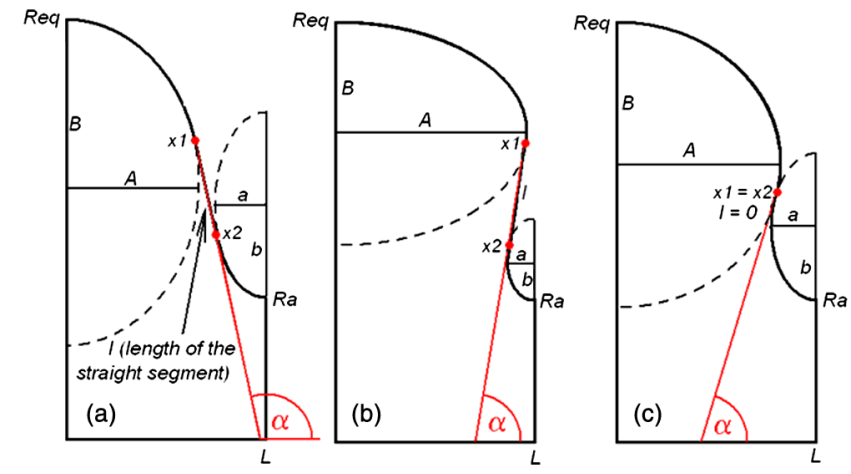

FIG. 1. Geometry of the elliptic half-cell: (a) nonreentrant, and (b),(c) reentrant.

The surface electric and magnetic fields in these cavities are shown in Figs. 3 and 4. One can see that the electric field has irregularities at the points $P$ and $Q$ on the surface of nonreentrant cavities-where the arc conjugates with the segment of the straight line. We believe that the best shape should have more regular dependencies for these curves. In the case of the reentrant cavity, the maximum is not flat as it is on nonreentrant geometry. One can suppose that flattening of this maximum will decrease the peak electric field but the $E_{\text {acc }}$ should not change significantly.

Comparing the magnetic fields of the TESLA cell and the reentrant cell with the same aperture we can see that decrease of the maximal field is obtained through the lengthening of the maximal field region. Again, better flattening of the maximal values of this field could decrease the peak field though not much. The area under these curves is approximately the same in both cases. In the case of the low-loss cavity the decrease of the field is obtained due to lesser aperture. As it was noted above, $H_{\mathrm{pk}} / E_{\mathrm{acc}}$ is a monotone function of the aperture radius and it is smaller here due to a smaller $R_{a}$.

Let us remind the reader that the record accelerating field obtained in the reentrant cavity [4] was achieved due to $10 \%$ lower magnetic peak field at the same $E_{\text {acc }}$ compared
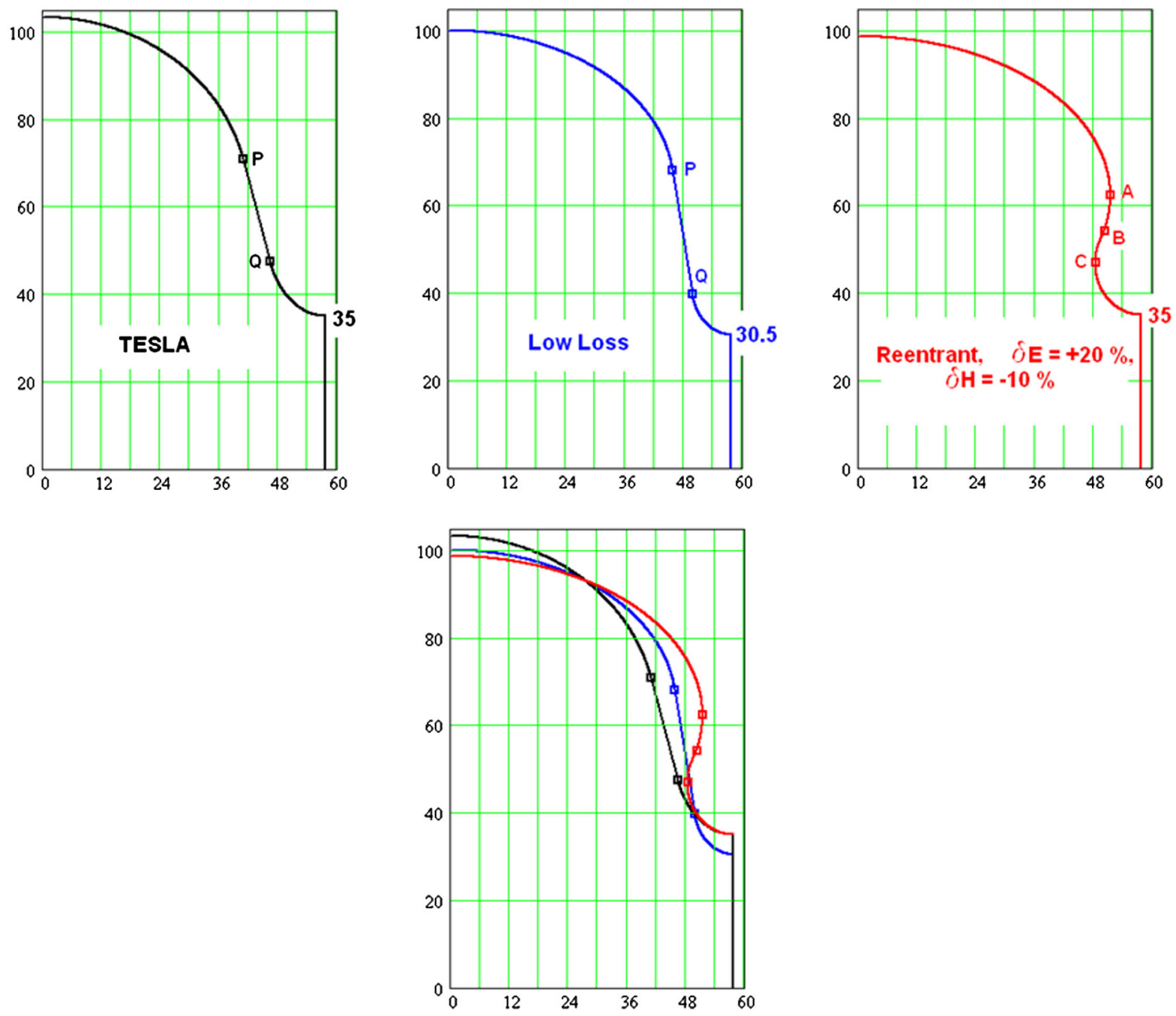

FIG. 2. Comparison of three known geometries of the elliptic half-cells. Dimensions are in mm. 


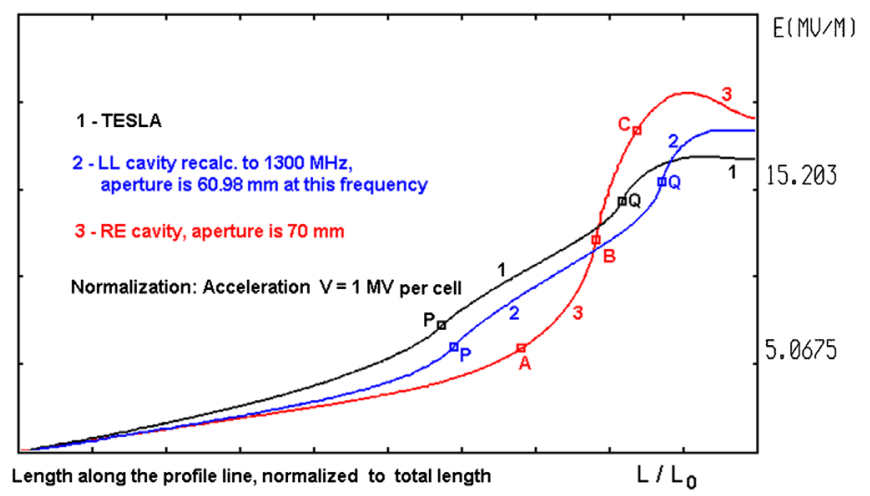

FIG. 3. Electric field on the surface of the cavities from Fig. 2.

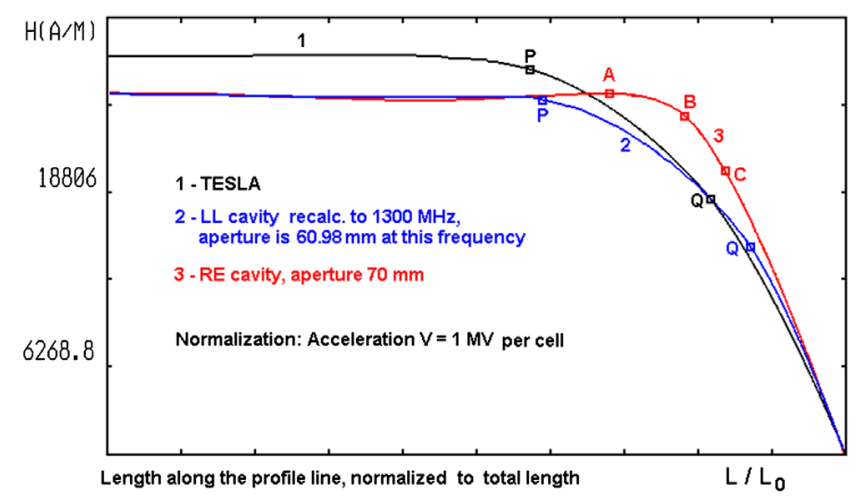

FIG. 4. Magnetic field on the surface of the cavities from Fig. 2.

to the TESLA geometry, though the value of $E_{\mathrm{pk}} / E_{\mathrm{acc}}$ was $20 \%$ higher.

Because of a high cost of the superconducting cavities even a decrease of further several percent of $H_{\mathrm{pk}} / E_{\mathrm{acc}}$ is worth the investigation, and we see that this decrease is possible. On the other hand we have an interesting problem to find the shape with a given distribution of fields on its surface.

\section{USAGE OF MAXWELL'S EQUATIONS}

The elliptic shape is a lucky choice because fields have nearly flat maxima and are multipactor resistant. However, it would be interesting to obtain the shape from the first principles, to have more regular field curves so we have an even better field flatness.

Using the first Maxwell's equation

$$
\oint_{l} H d l=\int_{s} \frac{\partial D}{\partial t} d s=\int_{s} \frac{\partial\left(\varepsilon_{0} E\right)}{\partial t} d s
$$

for the circular contours 1 and 2 (see Fig. 5) with surface fields $H_{s}$ and $E_{s}$ oscillating with a circular frequency $\omega$, we have

$$
\begin{aligned}
& \left(H_{s}+\Delta H_{s}\right) \cdot 2 \pi(R+\Delta R)-H_{s} \cdot 2 \pi R \\
& \quad=H_{s} \cdot 2 \pi \Delta R+\Delta H_{s} \cdot 2 \pi R=-\omega \varepsilon_{0} E_{s} 2 \pi R \Delta L .
\end{aligned}
$$

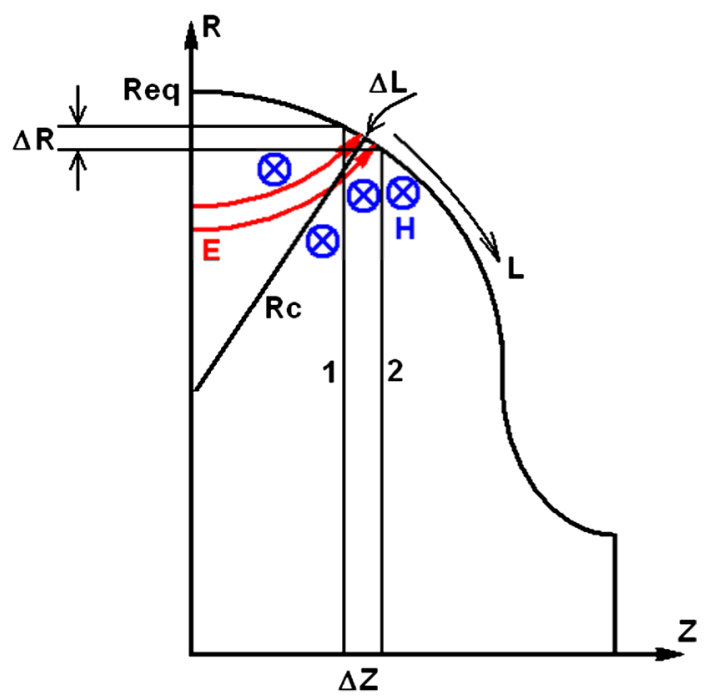

FIG. 5. Geometry for the first Maxwell's equation.

If $E_{s}$ is linear in $L, E_{s}=\alpha L$, see Fig. 3, the initial part of the curves $E$ vs $L$, and $H_{s}=$ const, that is $\Delta H_{s}=0$, then

$$
\frac{\Delta R}{R}=\frac{-\omega \varepsilon_{0} \alpha L}{H_{s}} \Delta L
$$

If $K=\omega \varepsilon_{0} \alpha / H_{s}=$ const, then

$$
R(L)=R_{\mathrm{eq}} \cdot e^{-K L^{2} / 2} .
$$

Further, $d Z=\sqrt{(d L)^{2}-(d R)^{2}}=\sqrt{1-(d R / d L)^{2}} d L$,

$$
Z(L)=\int_{0}^{L} \sqrt{1-R_{\mathrm{eq}}^{2} K^{2} l^{2} e^{-K l^{2}}} d l .
$$

This completes a parametric representation of $R$ and $Z$ as functions of $L$.

However, these simple approximations, $H_{s}=$ const, $E_{s}=\alpha L$, do not give an acceptable shape even for the upper part of the cavity contour line near the equator, Fig. 6 [10].

If we take into account dependencies of both $H_{s}$ and $E_{s}$ on the length along the profile line $L$ from the equator, we will have from (1):

$$
\frac{\Delta R}{R}=-\frac{\omega \varepsilon_{0} E_{s}}{H_{s}} \Delta L-\frac{\Delta H_{s}}{H_{s}} .
$$

Going from increments to derivatives and presenting $E_{s}$ and $H_{s}$ as functions of the coordinate along the profile line,

$$
E_{s}=e(L), \quad H_{s}=h(L),
$$

we will come to a differential equation

$$
\frac{1}{R} \frac{d R}{d L}=-\omega \varepsilon_{0} \frac{e(L)}{h(L)}-\frac{1}{h(L)} \frac{d h(L)}{d L}
$$




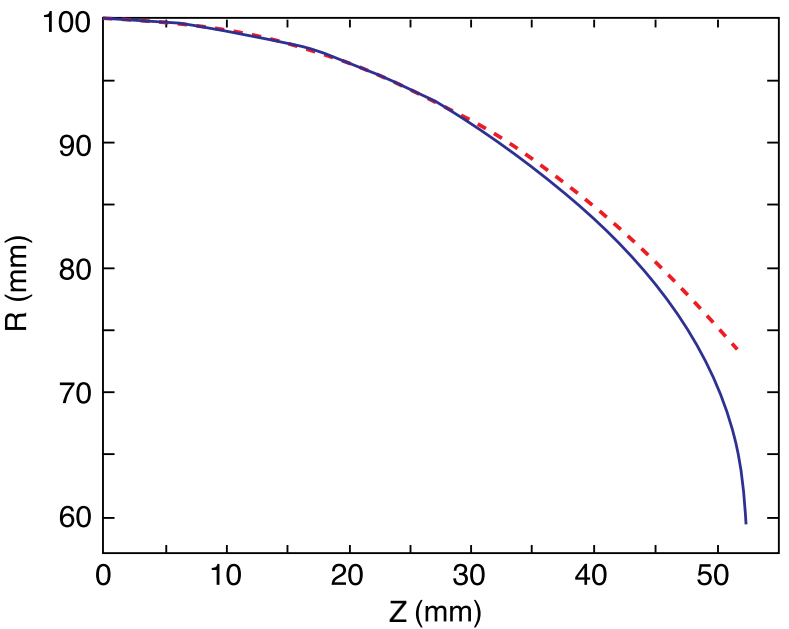

FIG. 6. The upper elliptic arc (solid blue line) and parametric curve (dashed red line) for the linear approximation for $E_{s}$.

that can be solved in quadratures:

$$
\begin{aligned}
R(L)= & R_{\mathrm{eq}} \cdot \exp \left[-\omega \varepsilon_{0} \int_{0}^{L} \frac{e(s)}{h(s)} d s\right. \\
& \left.-\int_{0}^{L} \frac{1}{h(s)}\left(\frac{d}{d s} h(s)\right) d s\right] \\
= & R_{\mathrm{eq}} \cdot \frac{h(0)}{h(L)} \cdot \exp \left[-\omega \varepsilon_{0} \int_{0}^{L} \frac{e(s)}{h(s)} d s\right], \\
Z(L)= & \int_{0}^{L} \sqrt{1-(d R(s) / d s)^{2}} d s .
\end{aligned}
$$

As it is shown at the beginning of this section, an arbitrary field distribution will not lead to a necessary shape: the solution should be self-consistent: fields define the shape, but the shape should define the same fields. Numerically found optimal shapes for the elliptic cavities have nearly flat fields on the equator $(H)$ and on the iris $(E)$. Further improvement of the shape is possible if we go beyond the paradigm of elliptic shapes.

\section{FIELDS ON THE SURFACE OF A PILLBOX CAVITY}

For an illustration, we will test the formulas (2) and (3) on the geometries with known surface fields.

Fields on the surface of a pill-box cavity (Fig. 7) can be presented in the form

$$
\begin{aligned}
& e(L)= \begin{cases}0, & L<a \\
E_{0} \cdot J_{0}(k r), & a<L<a+R_{\mathrm{eq}},\end{cases} \\
& h(L)= \begin{cases}\left(E_{0} / Z_{0}\right) \cdot J_{1}\left(\nu_{01}\right), & L<a \\
\left(E_{0} / Z_{0}\right) \cdot J_{1}(k r), & a<L<a+R_{\mathrm{eq}},\end{cases}
\end{aligned}
$$
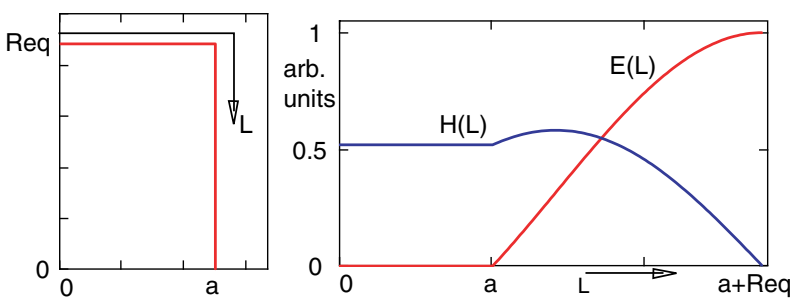

FIG. 7. Geometry: a pillbox half-cell with length $a$, and fields on its surface.

where $k=\omega / c, r=a+R_{\text {eq }}-L, J_{0}$, and $J_{1}$ are Bessel's functions of the first kind and $\nu_{01}$ is the first zero of $J_{0}(x)$.

Substituting these fields into (2) and (3) we have

$$
R(L)=R_{\text {eq }}, \quad Z(L)=L \quad \text { for } 0<L<a .
$$

For $a<L<a+R_{\text {eq }}$ we will have

$$
\begin{aligned}
R(L) & =R_{\mathrm{eq}} \frac{J_{1}(k r)}{J_{1}\left(\nu_{01}\right)} \cdot \exp \left[-\omega \varepsilon_{0} Z_{0} \int_{R_{\mathrm{eq}}}^{r} \frac{J_{0}(k s)}{J_{1}(k s)} d s\right] \\
& =R_{\mathrm{eq}} \frac{J_{1}(k r)}{J_{1}\left(\nu_{01}\right)} \cdot \exp \left[-\int_{k R_{\mathrm{eq}}}^{k r} \frac{J_{0}(x)}{J_{1}(x)} d x\right] \\
& =R_{\mathrm{eq}} \frac{J_{1}(k r)}{J_{1}\left(\nu_{01}\right)} \cdot \exp \left[\int_{k R_{\mathrm{eq}}}^{k r} \frac{J_{1}^{\prime}(x)+\frac{J_{1}(x)}{x}}{-J_{1}(x)} d x\right] \\
& =R_{\mathrm{eq}} \frac{J_{1}(k r)}{J_{1}\left(\nu_{01}\right)} \cdot \exp \left[-\ln J_{1}(x)-\ln x\right]_{k r}^{k R_{\mathrm{eq}}} \\
& =r=a+R_{\mathrm{eq}}-L, \\
Z(L) & =a .
\end{aligned}
$$

So, the formulas (2) and (3) proved correct for the pillbox cavity.

Analogously, the formulas were checked for a spherical cavity that also has an analytic solution for fields.

\section{TAYLOR EXPANSION OF THE FIELDS NEAR THE CAVITY IRIS}

We will analyze the electric and magnetic fields in the immediate vicinity of the iris tip, so we can suppose that they depend first of all on the radius of curvature $R_{c}$ at this point, not the lengths of the elliptic axes, Fig. 8. The aperture radius of the cavity is designated as $R_{a}$. We describe fields near the iris tip in the cylindrical system of coordinates: $\rho, \varphi, \zeta$. Let us present the fields in the form of series up to the third order of increments:

$$
\begin{aligned}
E_{\zeta}= & E_{0}\left[\alpha_{0} \zeta+\alpha_{1}\left(R_{a}-\rho\right) \zeta\right. \\
& \left.+\alpha_{2}\left(R_{a}-\rho\right)^{2} \zeta+\alpha_{3} \zeta^{3}\right], \\
E_{\rho}= & E_{0}\left[1+\beta_{0}\left(R_{a}-\rho\right)+\beta_{1}\left(R_{a}-\rho\right)^{2}\right. \\
& \left.+\beta_{2} \zeta^{2}+\beta_{3}\left(R_{a}-\rho\right)^{3}+\beta_{4}(R a-\rho) \zeta^{2}\right], \\
H_{\varphi}= & \omega \varepsilon_{0} E_{0}\left[a \zeta+b\left(R_{a}-\rho\right) \zeta+c\left(R_{a}-\rho\right)^{2} \zeta+d \zeta^{3}\right] .
\end{aligned}
$$




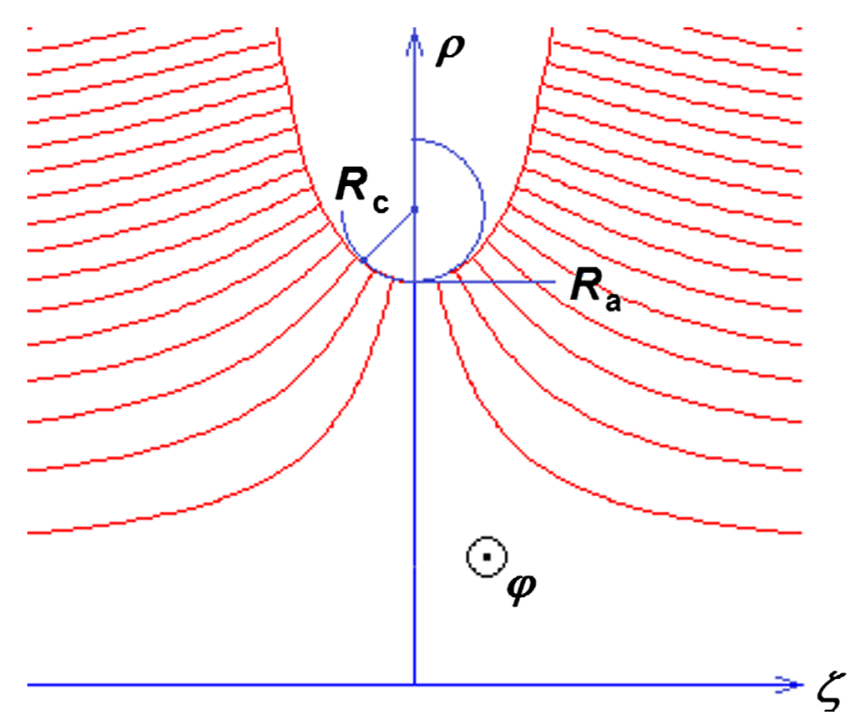

FIG. 8. Coordinate system and electric force lines near the iris.

Not all the terms of expansion with the power less than 4 are presented here because we take into account that $E_{\zeta}$ and $H_{\varphi}$ are odd functions of $\zeta$, and $E_{\rho}$ is an even function of this variable.

For calculation of the coefficients $\alpha_{0}, \ldots, \beta_{0}, \ldots, c, d$ we will use Maxwell's equations in the form

$$
\vec{\nabla} \times \vec{E}=-\partial \vec{B} / \partial t, \quad \vec{\nabla} \times \vec{H}=\partial \vec{D} / \partial t, \quad \vec{\nabla} \cdot \vec{E}=0
$$

with $\vec{B}=\mu_{0} \vec{H}$ and $\vec{D}=\varepsilon_{0} \vec{E}$. We will equate coefficients standing before equal terms of the expansion. For the correct comparison of coefficients, it is necessary that the polynomials for fields have equal powers for $R_{\mathrm{eq}}-\rho$ and $\zeta$ on both sides of the equations. This is why, when substituting fields (4) into the first formula from (5) we should take only linear and quadratic terms for $B_{\varphi}$, and in calculations with the second formula we should take a more precise expansion of $B_{\varphi}$ but only linear and quadratic terms for components of $\vec{E}$.

Further procedure of calculation of the coefficient is analogous to the procedure used for the equator region [7], and we omit it.

Finally, it appears that the number of equations is less by 2 than the number of unknown coefficients in (4). We can suppose that two of these coefficients can be found from additional consideration, for example from direct calculation of fields with SLANS [11]. It is convenient to find $\alpha_{3}$ and $\beta_{2}$ plotting $E_{\zeta}(\zeta)$ and $E_{\rho}(\zeta)$ versus $\zeta$ on the straight line $\rho=R_{a}$ when the terms with $R_{a}-\rho$ fall out; see (4).

Some coefficients can be found immediately from the local geometry, i.e. they depend on $R_{a}$ and $R_{c}$, one of them is just a constant:

$$
\alpha_{0}=-\frac{1}{R_{c}}, \quad \beta_{0}=\frac{1}{R_{a}}-\frac{1}{R_{c}}, \quad b=-\beta_{0}, \quad a=-1 .
$$

As will be shown below, $\alpha_{3}$ and $\beta_{2}$ depend on the remote geometry: equatorial radius $R_{\text {eq }}$ and the equatorial elliptic arc half-axes. Other coefficients can be expressed in terms of the previous ones:

$$
\begin{aligned}
\beta_{4} & =\beta_{2} / R_{a}^{2}+3 \alpha_{3}, \\
d & =-\beta_{2} / 3 \\
\alpha_{1} & =-k^{2}-2 \beta_{2}, \\
\alpha_{2} & =\frac{k^{2}}{2}\left(\frac{1}{R_{c}}-\frac{1}{R_{a}}\right)-\beta_{4}, \\
c & =-\frac{\alpha_{1}}{2}+\frac{1}{2 R_{a} R_{c}}-\frac{1}{R_{a}^{2}}, \\
\beta_{1} & =-c \\
\beta_{3} & =\frac{1}{3}\left(\frac{\beta_{1}}{R_{a}}+\frac{2}{R_{a}^{3}}-\frac{1}{R_{a}^{2} R_{c}}+\alpha_{2}\right) .
\end{aligned}
$$

\section{DEPENDENCE OF THE LOCAL SURFACE FIELDS ON THE REMOTE GEOMETRY}

\section{A. Electric field on the iris}

The surface electric field on the iris, accurate to the second order of $\zeta$ does not depend on $\alpha_{3}$ but depends on $\beta_{2}$ :

$$
E_{s}(\zeta)=E_{0}\left[1+\left(\frac{1}{R_{c}^{2}}-\frac{1}{2 R_{a} R_{c}}+\beta_{2}\right) \zeta^{2}\right]
$$

Up to the second order of the expansion, the flat field should have a zero multiplier before $\zeta^{2}$ in (6).

In the construction of a flat electric field on the surface near the iris tip, it appears necessary to take into account the remote geometry: having the same frequency $\omega$ and $R_{a}$ and $R_{c}$ on the iris but different $R_{\mathrm{eq}}$ and half-axes of the upper arc we will have different behavior of the surface electric field on the iris as it is shown in Fig. 9.

The value of $1 / R_{c}^{2}-1 /\left(2 R_{a} R_{c}\right)=15520 \mathrm{~mm}^{-2}$ in this example is close to the values of $\beta_{2}$ but has a different sign, so the coefficient before $\zeta^{2}$ changes much though values of $\beta_{2}$ are close in both cases.

\section{B. Magnetic field in the equator area}

Expansion of the field in the equatorial area was done earlier [7] where it was used for calculations of multipactor. In that case, analogously to the case of the electric field near the iris, it appeared that the number of equations was not sufficient to find all the terms of the expansion to the second order. We introduced the parameter $\nu$ that, analogously to $\beta_{2}$, can be changed when the remote parts of the geometry are changed.

In the construction of a flat magnetic field near the equator, on the upper arc, we first supposed that the field behavior depends mainly on the local geometry and can be corrected by moving different parts of this arc relative to 

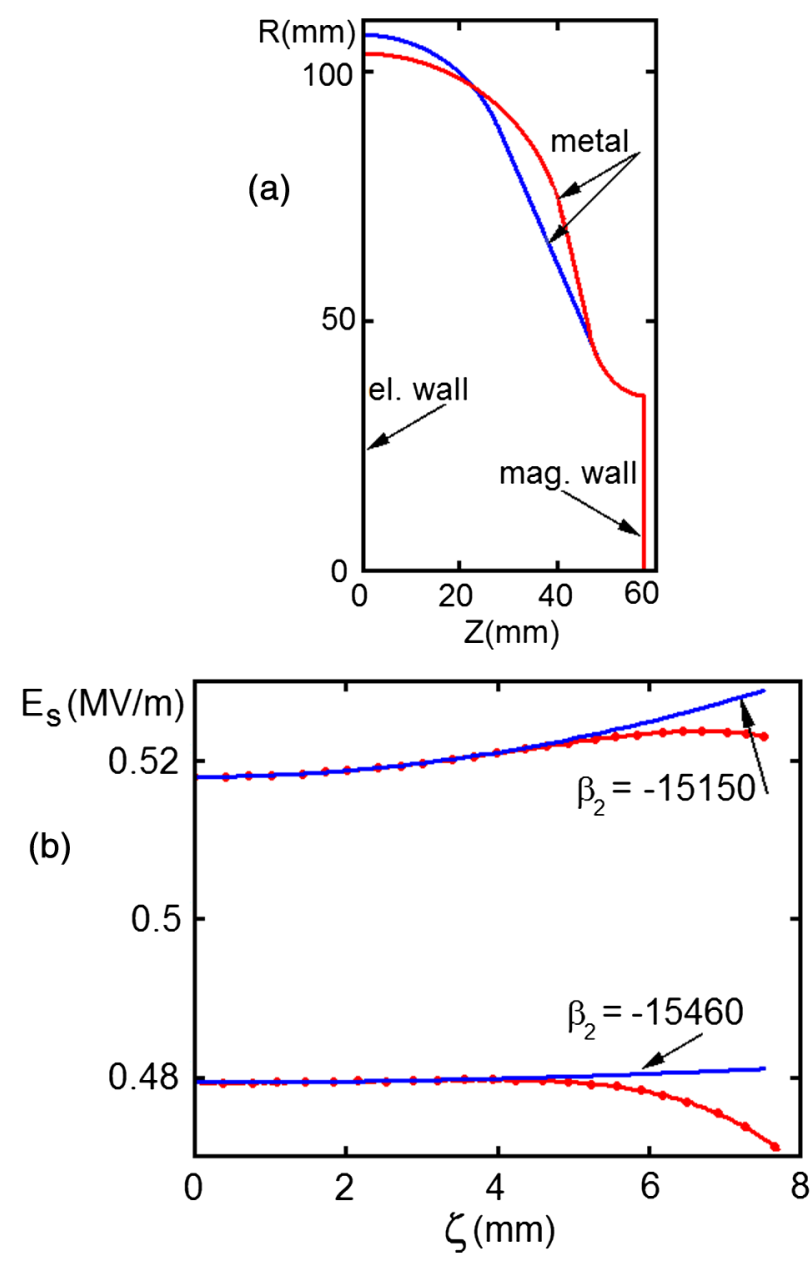

FIG. 9. Example of two cavities (a) with equal frequencies, equal $R_{a}$, equal radii of curvature at the iris but having different $\beta_{2}$ and different behavior of the electric field on the iris (b).

others. However, it appeared wrong, and an example shown in Fig. 10 confirms this statement.

For the cavities shown in Fig. 10(a), the magnetic field on the initial part of the surface is shown in Fig. 10(b). Also shown are the parabolic approximations of these fields in the form $y(Z)=H_{1} \cdot\left(1-\nu \cdot Z^{2} /\left(2 R_{c}^{2}\right)\right)$. Normalization to $2 R_{c}^{2}$ is used for a comparison with the spheric cavity which has $\nu=1$.

We conclude that the behavior of fields on the upper arc depends not on the geometry of this arc only but also on the geometry of the iris area. It can be impossible to make a flat field on the upper arc by changing this arc only.

\section{CONSISTENCY OF FIELDS ON THE UPPER ARC WITH ITS SHAPE}

In [7], the surface fields near the equator on the upper arc of the cavity profile were presented in the form of expansions. Here we will restrict ourselves to the second power of these expansions, the next term for the electric field $E_{s}$ is cubic:
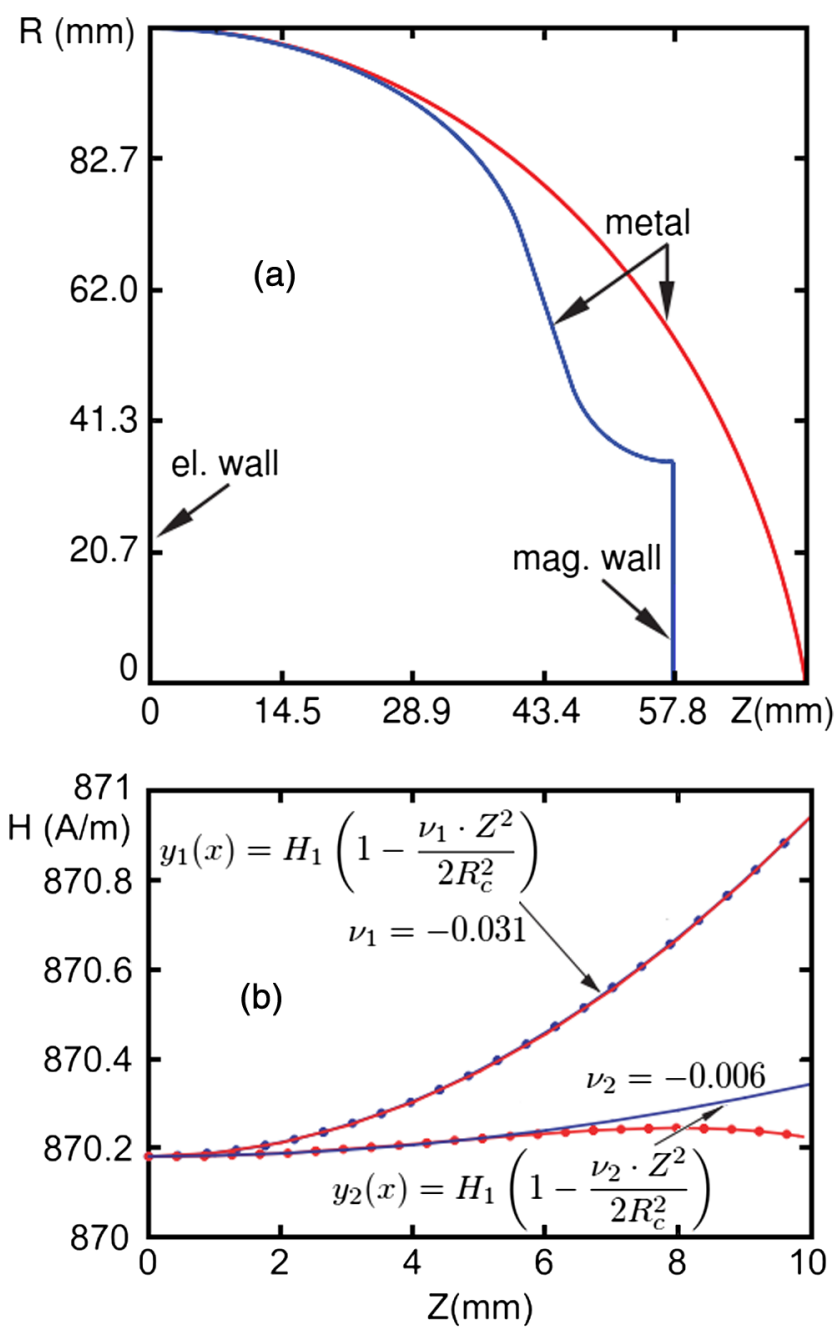

FIG. 10. Example of 2 cavities (a) with equal frequencies, equal $R_{\text {eq }}$, equal radii of curvature at the equator but different behavior of the magnetic fields near the equator (b). The curve $H(Z)$ with $\nu=-0.031$ is for the TESLA inner cell.

$$
\begin{aligned}
& E_{s}=\omega B_{0} \beta_{0} \zeta, \\
& B_{s}=B_{0}\left[1+a\left(R_{\mathrm{eq}}-\rho\right)+h \zeta^{2}\right] .
\end{aligned}
$$

The coefficients of this expansion are as follows [7]:

$$
\begin{aligned}
a & =1 / R_{\mathrm{eq}}, \\
h & =-a / 2 R_{c}-\nu / 2 R_{c}^{2}, \\
\beta_{0} & =-2 h / k^{2} .
\end{aligned}
$$

The coordinate system and fields are shown in Fig. 11. To the second order of accuracy, we consider the arc of the upper ellipse as an arc of a circle with the same curvature as the ellipse: $R_{c}=A^{2} / B$, where $A$ and $B$ are half-axes of the ellipse. So we have for the points of the arc

$$
R_{\mathrm{eq}}-\rho=\zeta^{2} / 2 R_{c}
$$




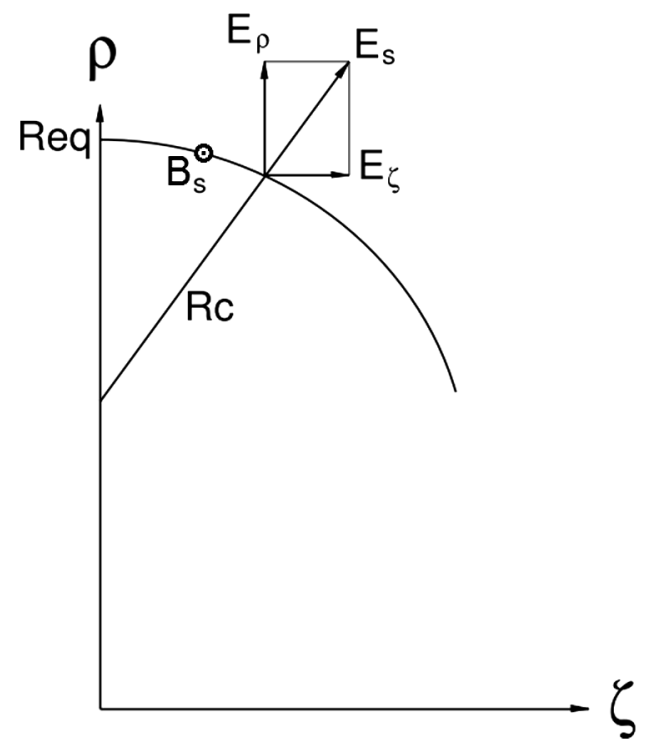

FIG. 11. Coordinate system for the fields on the upper arc.

Using (8) and (9) we can rewrite (7):

$$
\begin{aligned}
E_{s} & =\frac{\omega B_{0}}{k^{2} R_{c}}\left(\frac{1}{R_{\mathrm{eq}}}+\frac{\nu}{R_{c}}\right) \zeta, \\
H_{s} & =\frac{B_{0}}{\mu_{0}}\left(1-\frac{\nu}{2 R_{c}^{2}} \zeta^{2}\right) .
\end{aligned}
$$

So, fields on the upper arc near the equator depend on the parameter $\nu$ that depends on the geometry of the lower part of the cavity as it was shown in the previous section. On the other hand, the geometry of the upper arc $\left(R_{\text {eq }}\right.$ and $\left.R_{c}\right)$ should not depend on this parameter because this geometry was defined earlier for these fields.

To resolve this issue, let us substitute Eq. (7) into (2). We have

$$
\begin{aligned}
R(L)= & \frac{R_{\mathrm{eq}}}{1-\frac{\nu}{2 R_{c}^{2}} \cdot L^{2}} \\
& \times \exp \left[-\int_{0}^{L} \frac{1}{R_{c}}\left(\frac{1}{R_{\mathrm{eq}}}+\frac{\nu}{R_{c}}\right) \frac{\zeta d \zeta}{1-\frac{\nu}{2 R_{c}^{2}} \zeta^{2}}\right] \\
= & R_{\mathrm{eq}} \cdot\left(1-\frac{\nu L^{2}}{2 R_{c}^{2}}\right)^{\frac{R_{c}}{\nu R_{\mathrm{eq}}}} \approx R_{\mathrm{eq}}-\frac{L^{2}}{2 R_{c}} .
\end{aligned}
$$

One can see that (11) coincides with (9) within an accuracy of designations, so that fields with different values of $\nu$ correspond to the same geometrical parameters of the upper arc.

Regretfully, integrals analogous to (11) for the iris area are not so simple, and we did not find an expression for this case.

\section{CONCLUSION}

Relationships between surface electric and magnetic field on one side and the shape of a hollow axially symmetric accelerating cavity on the other side are found. Several applications of this formula are demonstrated. Taylor expansions of the fields near the equator and the iris tip are presented. It is shown that a cavity shape with better parameters of $E_{\mathrm{pk}} / E_{\mathrm{acc}}$ and $H_{\mathrm{pk}} / E_{\mathrm{acc}}$ cannot be constructed in the elliptic family of geometries and some deviations from the elliptic curves have to be analyzed. This work is now in progress.

\section{ACKNOWLEDGMENTS}

Authors are thankful to Jeanne Butler for help. This work was supported by NSF Grant No. DE-SC0010564.

[1] Hasan Padamsee et al., RF Superconductivity for Accelerators (John Wiley \& Sons, New York, 1998).

[2] P. Kneisel, R. Vincon, and J. Halbritter, Nucl. Instrum. Methods Phys. Res. 188, 669 (1981).

[3] V. Shemelin, H. Padamsee, and R. L. Geng, Nucl. Instrum. Methods Phys. Res., Sect. A 496, 1 (2003).

[4] Rong-Li Geng, Hasan Padamsee, Andrew Seaman, and Valery Shemelin, in Proceedings of the 21st Particle Accelerator Conference, Knoxville, TN, 2005 (IEEE, Piscataway, NJ, 2005).

[5] Valery Shemelin, Phys. Rev. ST Accel. Beams 12, 114701 (2009).

[6] Valery Shemelin, Cornell University, LEPP Report No. SRF070614-02, 2007.

[7] Valery Shemelin, Phys. Rev. ST Accel. Beams 16, 012002 (2013).

[8] B. Aune et al., Phys. Rev. ST Accel. Beams 3, 092001 (2000).

[9] J. Sekutowicz, P. Kneisel, G. Giovati, and H. Wang, Report No. JLab-TN-02-023, 2002.

[10] D. Stark and V. Shemelin, Cornell University, LEPP Report No. 090814-01, 2009.

[11] "The SuperLANS Family of Codes for 2D RF Cavity Simulations," www.euclidtechlabs.com/SLANS/slans.php. 